\title{
Five-Year Clinical Outcomes of Trabectome Surgery at a Single Glaucoma Center
}

Hamed Esfandiari, ${ }^{1,2}$ Priyal Shah, ${ }^{1}$ Pooya Torkian, ${ }^{2}$ Ian P. Conner, ${ }^{1}$ Joel S. Schuman, ${ }^{3}$ Kiana Hassanpour, ${ }^{2}$ Nils

$$
\text { A. Loewen }{ }^{1 *}
$$

${ }^{1}$ Department of Ophthalmology, School of Medicine, University of Pittsburgh, Pittsburgh, Pennsylvania, United States

${ }^{2}$ Ophthalmic Research Center, Shahid Beheshti University of Medical Sciences, Tehran, Iran

${ }^{3}$ Department of Ophthalmology, School of Medicine, New York University, New York City, New York, United States

*Correspondence to Nils Loewen, MD, PhD

203 Lothrop St, Suite 819, Pittsburgh, PA 15213

Department of Ophthalmology, School of Medicine, University of Pittsburgh, Pittsburgh, Pennsylvania, United States.

Email: loewen.nils@gmail.com 


\section{Abstract}

Purpose: To analyze the five-year results of Trabectome ab interno trabeculectomy of a single glaucoma center.

Method: In this retrospective interventional single-center case series, data of 93 patients undergoing ab interno trabeculotomy between September 2010, and December 2012 were included. Kaplan-Meier analysis was performed using success criteria defined as postoperative intraocular pressure (IOP) $\leq 21 \mathrm{~mm}$ $\mathrm{Hg}$, or $>20 \%$ reduction from preoperative IOP, and no need for further glaucoma surgery. Risk factors for failure were identified using Cox proportional hazards ratio (HR).

Results: The retention rate for five years follow-up was $66 \%$. The cumulative probability of success at 1,2 , 3, 4 and 5 years was $82.6 \%, 76.7 \%, 73.9 \%, 72.3 \%$, and $67.5 \%$. Risk factors for failure were lower baseline IOP $(H R=0.27, P=0.001)$, younger age $(H R=0.25, P=0.02)$, and higher central corneal thickness $(H R=0.18, P=$ 0.01). Pseudoexfoliation was associated with a higher success rate $(H R=0.39, P=0.02)$. IOP was decreased significantly from $20.0 \pm 5.6 \mathrm{mmHg}$ at baseline to $15.6 \pm 4.6 \mathrm{mmHg}$ at 5 -year follow-up $(\mathrm{P}=0.001)$. The baseline number of glaucoma medications was 1.8 \pm 1.2 , which decreased to $1.0 \pm 1.2$ medications at 5 years. Conclusion: Trabectome surgery was associated with a good long-term efficacy and safety profile in this single-center case series with a high retention rate. A higher baseline IOP, older age, thinner cornea, and pseudoexfoliation glaucoma were associated with a higher success rate.

Keywords: Ab interno trabeculectomy; Trabectome surgery; long-term outcomes; microinvasive glaucoma surgeries; MIGS 


\section{Introduction}

Management of glaucoma has undergone significant changes with a trend towards earlier microincisional glaucoma surgery (MIGS) instead of filtering surgery later in the course of the disease. Ab interno trabeculectomy (AIT) with the Trabectome (NeoMedix Corp., Tustin, CA, USA) was among the first MIGS procedures and ablates the trabecular meshwork and inner wall of Schlemm's canal (SC), the primary sites of outflow resistance in the pathophysiology of glaucoma [1]. The Trabectome surgical system creates a high-frequency alternating current at the tip of the handpiece to molecularize and aspirate the trabecular meshwork without dragging adjacent structures [2,3]. The released energy is limited to the space between the two electrodes, and the outer wall of SC is shielded by a footplate. The collapsible nature of the plasma bubble is akin to a disruptive laser and prevents thermal damage to the adjacent tissue. This process is different from cautery that generates heat to coagulate tissue, as occasionally mischaracterized.

Trabectome surgery has a nearly 15 year track record of use in different types of glaucoma including pseudoexfoliation [4], inflammatory [5], pigmentary [6], steroid induced [7], angle-closure glaucoma [8, 9], and complex glaucomas that have led to failure of other surgeries $[10,11]$.

Many of the reported case series involve a database that the manufacturer is required to maintain as part of a post-market surveillance, and it includes the first 20 surgeries when new Trabectome surgeons become certified $[1,12-14]$. The most recent update on this dataset reported a $26 \%$ reduction of IOP from the baseline 7.5 years after surgery with a success rate of $60 \%$ on 1.6 drops [14]. While useful, this data may be biased by reporting more data of less experienced surgeons and have limited follow up and patient retention [15]. The largest and most recent study of these started with 5435 eyes, but only 37 remained for the final analysis [14].

The purpose of our study was to report the long-term outcomes of Trabectome procedure in a cohort of patients in a single glaucoma center with a high retention rate. 


\section{Methods}

An Institutional Review Board approval was obtained from the University of Pittsburgh Human Subjects Research Committee. We followed the tenets of the Declaration of Helsinki and regulations of the Health Insurance Portability and Accountability Act. Patients who underwent Trabectome surgery at our glaucoma center between September 2010 and December 2012 were identified using current procedural terminology (CPT) codes and included in the study. Patients were included regardless of same-session phacoemulsification because the impact of same-session phacoemulsification on IOP is negligible in Trabectome surgery $[16,17]$.

Exclusion criteria were a history of incisional and angle surgeries, combined glaucoma surgeries, and less than five years follow-up. Information collected included demographic data, types of glaucoma, preand postoperative intraocular pressures (IOP), baseline ocular biometric characteristics including axial length $(A L)$, central corneal thickness (CCT), anterior chamber depth (ACD), lens thickness (LT), number of pre-and postoperative glaucoma medications, visual field mean deviation (MD), visual field index (VFI), type of surgery, and intra- and postoperative complications. Glaucoma severity was based on the Advanced Glaucoma Intervention Study (AGIS) system [18]. At each postoperative visit, BCVA, IOP, glaucoma medications, and complications were noted.

The primary outcome measure was success and defined as $5<10 P \leq 21 \mathrm{mmHg}, \geq 20 \%$ reduction of IOP from baseline at two consecutive visits, no need for further glaucoma surgery, and no loss of light perception. Qualified success was defined as achieving success with or without medications. Patients who achieved success without medication were labeled as complete success. A Kaplan Meier survival analysis was used to evaluate the success rates. The secondary outcome measures were IOP, glaucoma medications, and complications. Risk factors for success and failure were identified using the Cox proportional hazards ratio (HR) model with multivariate analysis.

\section{Surgical technique}

Trabectome surgery was performed first in cases where a phacoemulsification and lens implantation was performed in the same surgery. The patient's head was rotated away from the surgeon by $30^{\circ}$ while the microscope was tilted towards the surgeon by the same amount. A temporal $1.6 \mathrm{~mm}$ incision was made 2 $\mathrm{mm}$ anterior to the limbus and parallel to the iris plane. The Trabectome was inserted through the incision and positioned across the AC at the nasal angle. The tip of the Trabectome was inserted into Schlemm's canal and engaged with the TM. The aspiration and ablation were activated with the power set to 0.8 to 1 
watts. The same was done in the opposite direction to remove the TM over approximately $160^{\circ}$ total. The tip was withdrawn from the anterior chamber, and the incision was hydrated. All glaucoma medications were discontinued after the surgery and resumed if the IOP was not within the target range. All patients received pilocarpine four times a day for one month followed by three times a day for a second month, a practice recently demonstrated to be unnecessary [8].

\section{Statistical analysis}

All analyses were performed using SPSS software (SPSS Statistics for Windows, Version 25, Armonk, NY, IBM Corporation). Frequency, percent, mean $\pm S D$, median, and range were used to describe the data. To compare the change in IOP and number of medications, we used an interaction analysis within a linear mixed model. To evaluate the baseline differences between groups, we used the T-test, Chi-Square, and Fisher's exact test. Kaplan-Meier survival plots were constructed to assess the long-term survival rates and compared using the log-rank test. A Cox proportional Hazard model was used to find risk factors for failure and to estimate the adjusted hazard ratio of each factor. Statistical significance was set at $p<0.05$. Success was defined as IOP $<21 \mathrm{mmHg}$ and a $>20 \%$ reduction from baseline with no need for additional glaucoma surgery.

\section{Results}

Patients typically had open angle glaucoma refractory to medical therapy, or they had visually significant cataracts in the setting of glaucoma on medical therapy. Ninety-three eyes underwent AIT during this period of which 32 were excluded for the following reasons: four (12.5\%) individuals died before the 5-year visit, 18 (56\%) eyes had AIT combined with a Baerveldt glaucoma implant [19], four (9.3\%) eyes had a complicated cataract surgery after AIT, and seven (21.8\%) eyes were resident cases. All patients were phakic at the time of surgery and phacoemulsification was combined with AIT in all cases.

Table 1 presents baseline demographics. The mean age of study participants was $71.9 \pm 10.2$ years, and $50.8 \%$ of the patients were female $(P=0.911)$. Primary open-angle glaucoma (POAG) was the most common diagnosis (62.3\%) followed by exfoliation glaucoma (XFG, 14.8\%), primary angle closure glaucoma (PACG, 8.2\%), pigmentary glaucoma (PG, 6.6\%), and others that included steroid-induced or anti-VEGF agent related glaucoma (8.2\%). The baseline glaucoma severity was characterized based on the AGIS system[18] was mild in (34.4\%), moderate (26.3\%), and severe (39.9\%) of eyes. 


\section{Surgical Success and risk factors for failure}

The Kaplan-Meier survival curves (Figure 1) indicated a mean survival of $49.8 \pm 2.8$ months. The cumulative probability of success at $1,2,3,4$ and 5 years was $82.6 \%, 76.7 \%, 73.9 \%, 72.3 \%$ and $67.5 \%$. Corresponding values for the POAG subgroup was $83.7 \%, 78.3 \%, 76.7 \%, 70.1 \%$ and $65.1 \%$.

The multivariate Cox regression model was stratified by age, gender, baseline IOP, number of medications, glaucoma type, central corneal thickness (CCT), anterior chamber depth (AC depth), lens thickness, axial length, Humphrey visual field mean deviation (HVF MD) and Humphrey visual field index (HVF VFI). The risk factors for failure were a lower baseline IOP $(H R=0.27, P=0.001)$, younger age $(H R=0.25$, $\mathrm{P}=0.02)$, and a higher CCT $(H R=0.18, P=0.01)$. Diagnosis of $X F G(H R=0.39, P=0.02)$ was associated with higher chance of success at final follow up (Table 2).

\section{Intraocular pressure}

IOP was decreased significantly from $20 \pm 5.6 \mathrm{mmHg}$ at baseline to $15.6 \pm 4.6 \mathrm{mmHg}$ at the 5-year follow-up $(P=0.001)$. The mean IOP at $1,2,3$ and 4 th year was $13.6 \pm 3.4,14.3 \pm 3.9,14.5 \pm 3.9$ and $14.7 \pm 3.8$. $(P<0.001)$ IOP remained unchanged six months after the surgery (Figure 2). The baseline number of glaucoma medications was $1.84 \pm 1.15$. Patients received $0.79 \pm 1.14,1.03 \pm 1.15,1.07 \pm 1.3,1.09 \pm 1.23$, and $1.01 \pm 1.15$ medications at 1, 2, 3, 4, and 5 years follow up. However, only the difference between the first year and the baseline was statistically significant $(P=0.006)$.

\section{Best corrected visual acuity}

The mean BCVA at the baseline was $0.56 \pm 0.61 \log M A R$, which changed to $0.42 \pm 0.65 \log M A R$ at final visit $(p=0.40)$. Only two patients lost more than two lines of visual acuity.

\section{Reoperation for glaucoma}

Subsequent glaucoma surgery was done when the IOP could not be controlled within the individual target range or when there was evidence of disease progression. Additional glaucoma surgeries included two Ahmed glaucoma valve (AGV) implantations, one trabeculectomy with MMC, and one cyclophotocoagulation.

\section{Postoperative observations and complications}

The most common observation was a postoperative microhyphema or layered hyphema on postoperative day 1 that occurred in $48.4 \%$. The mean duration was $11.7 \pm 10.0$ days. Late-onset hyphema, defined as occurring more than two months postoperatively, was noted in 4.9\% of eyes and ranged from 64 to 634 
days after the surgery. Temporary postoperative IOP surges of more than $10 \mathrm{mmHg}$ were observed in $27.4 \%$ of patients, and almost all them occurred within the first month. No serious vision-threatening complications occurred.

\section{Discussion}

Trabectome surgery was effective in reducing the IOP and number of glaucoma medications at the 5-year follow-up visit. The success rate at five years was $68 \%$, higher than in case series derived from the manufacturer maintained dataset [1, 12-14]. The main reason for failure was uncontrolled IOP. A possible explanation for a higher success rate in our study is that all surgeries were performed by a more experienced surgeon. On average, new surgeons need at least five eyes to become comfortable and safe with the procedure [20], but it takes almost 30 eyes to consistently maximize outflow [20], well beyond the initial 20 cases included the manufacturer dataset. It is still possible that our study underestimates current success rates because the device had only been introduced in our clinic in 2009. Additionally, many patients who reside in more remote locations with successful outcomes can be managed by their primary eye care provider and do not always follow up with the surgeon unless the necessity arises.

On average, the IOP was lowered by approximately $20 \%$ to near $15 \mathrm{~mm} \mathrm{Hg}$ while decreasing medications by less than one, with a low rate of serious complications. As seen in previous reports, IOP remained mostly stable six months postoperatively [13, 21, 22]. Two patients in our study lost two lines of visual acuity during the five years observed and had advanced glaucoma with splitting fixation with a baseline HVF MD of -18.23 and $-23.7 \mathrm{~dB}$. Phacoemulsification combined with AIT does not in itself cause a change of the refractive outcomes when compared to phacoemulsification alone [23]. Ahuja et al. noted that 5\% of patients in their series lost more than two lines of Snellen visual acuity, but a cause was not provided [24].

The multivariate analysis of risk factors showed that patients with pseudoexfoliation glaucoma have a $36 \%$ higher chance of success in comparison to POAG reflecting the experience of other investigators [24-27]. Similar to other secondary open angle glaucomas that primarily affect the trabecular meshwork [5, 7, 28], exfoliation glaucoma responds well to trabecular ablation that removes the diseased tissues while at the same time one of the primary sources of the pseudoexfoliative material, the lens, is removed in cataract surgery. Our results also match the previously reported findings that a higher baseline IOP is associated with higher IOP reduction $[13,22,27]$. 
We observed an association between a thicker CCT and risk of failure. CCT is reflecting the corneal and scleral biomechanical properties, in particular, corneal rigidity [29-32]. After trabecular ablation, outflow is only limited by the remaining downstream outflow resistance in the outer wall of Schlemm's canal, in collector channels and episcleral veins. These channels have features that indicate that they may be able to regulate the outflow resistance [33-35]. More research is needed to clarify the role of corneal biomechanics in the outcome of angle surgeries.

Our study was limited by its retrospective nature and a relatively low number of patients which is the result of only including patients with full, 5-year datasets. All patients underwent Trabectome surgery combined with cataract extraction which we have shown does not affect the IOP reduction $[12,16,22]$ but may create a bias towards milder glaucomas at baseline. This study was conducted at a single tertiary academic referral center, and the results cannot easily be generalized to other practice facilities.

In summary, this single-center case series had a high patient retention rate and showed a high long-term safety and efficacy of Trabectome surgery. Risk factors for failure were a lower baseline IOP, younger age, and higher central corneal thickness while pseudoexfoliation glaucoma increased the chances of success.

\section{Funding}

We acknowledge support from The Initiative to Cure Glaucoma, The Eye and Ear Foundation of Pittsburgh; NIH CORE Grant P30 EY08098 to the Department of Ophthalmology; from an unrestricted grant from Research to Prevent Blindness, New York, NY.

\section{Conflict of interest}

NAL has received honoraria for Trabectome wet labs and lectures from Neomedix Corp.

All procedures performed in studies involving human participants were in accordance with the ethical standards of the institutional and/or national research committee and with the 1964 Helsinki declaration and its later amendments or comparable ethical standards.

For this type of study formal consent was not required. 


\section{Tables}

\section{Table 1}

Table 1: Baseline characteristic.

\begin{tabular}{|c|c|c|}
\hline Variable & & Value \\
\hline Age (years) & Mean $\pm S D$ & $71.9 \pm 10.2$ \\
\hline \multirow[t]{2}{*}{ Gender } & $\mathrm{F}$ & $31(50.8 \%)$ \\
\hline & M & $30(49.2 \%)$ \\
\hline \multirow[t]{2}{*}{ Eye } & Right & $34(55.7 \%)$ \\
\hline & Left & 27 (44.3\%) \\
\hline Axial Length & Mean $\pm S D$ & $24 \pm 1.5$ \\
\hline \multirow[t]{2}{*}{$\mathrm{CCT}$} & Mean \pm SD & $548 \pm 37$ \\
\hline & Median (range) & 547 (445 to 606$)$ \\
\hline \multirow[t]{2}{*}{ AC Depth } & Mean $\pm S D$ & $3.35 \pm 0.68$ \\
\hline & Median (range) & 3.24 (2 to 5.03 ) \\
\hline Lens Thickness & Mean $\pm S D$ & $4.23 \pm 1.22$ \\
\hline HVF MD & Mean $\pm S D$ & $-7.5 \pm 8.9$ \\
\hline \multirow[t]{2}{*}{ HVF VFI } & Mean \pm SD & $79 \pm 28$ \\
\hline & Median (range) & 94 (2 to 100$)$ \\
\hline $\log M A R$ & Mean $\pm S D$ & $0.45 \pm 0.55$ \\
\hline \multirow[t]{2}{*}{ Baseline IOP } & Mean \pm SD & $20 \pm 5.6$ \\
\hline & Median (range) & 18 (9 to 30$)$ \\
\hline \multirow[t]{2}{*}{ Baseline medications } & Mean \pm SD & $1.84 \pm 1.15$ \\
\hline & Median (range) & 1 (0 to 4$)$ \\
\hline \multirow[t]{5}{*}{ Glaucoma Type } & POAG & $38(62.3 \%)$ \\
\hline & XFG & $9(14.8 \%)$ \\
\hline & CACG & $5(8.2 \%)$ \\
\hline & PG & $4(6.6 \%)$ \\
\hline & Mixed Mechanism & $5(8.2 \%)$ \\
\hline \multirow[t]{3}{*}{ Glaucoma AGIS Stage } & Mild & $21(34.4 \%)$ \\
\hline & Moderate & $16(26.3 \%)$ \\
\hline & Severe & $24(39.3 \%)$ \\
\hline
\end{tabular}

CCT: central corneal thickness, AC: anterior chamber, HVF: Humphrey visual field, MD: mean deviation, VFI: visual field index, IOP: intraocular pressure, POAG: primary open angle glaucoma, XFG: exfoliation glaucoma, CACG: Chronic angle closure glaucoma, PG: pigmentary glaucoma, AGIS: Advanced Glaucoma Intervention Study. 


\section{Table 2}

Table2. Cox proportional hazard regression model.

\begin{tabular}{|l|l|l|l|}
\hline & & Hazard Ratio & P-value \\
\hline Gender & & $0.851(0.458-1.65)$ & 0.622 \\
\hline XFG & & $0.39(0.29-0.46)$ & 0.02 \\
\hline Lens Thickness & & $0.83(0.66-1.05)$ & 0.13 \\
\hline Axial Length & & $1.01(0.8-1.25)$ & 0.57 \\
\hline Baseline BCVA & & $0.87(0.54-1.4)$ & 0.93 \\
\hline Baseline Medications & 1 medication & $0.46(0.107-1.98)$ & 0.29 \\
\hline & 2 medications & $0.82(0.26-2.53)$ & 0.73 \\
\hline Baseline IOP & 3 medications & $0.79(0.79-3.05$ & 0.73 \\
\hline & 4 medications & $0.52(0.52-5.8)$ & 0.60 \\
\hline & & $0.27(0.13-0.59)$ & 0.001 \\
\hline
\end{tabular}

XFG: pseudoexfoliation glaucoma, BCVA: best corrected visual acuity, IOP: intraocular pressure 
Figure 1
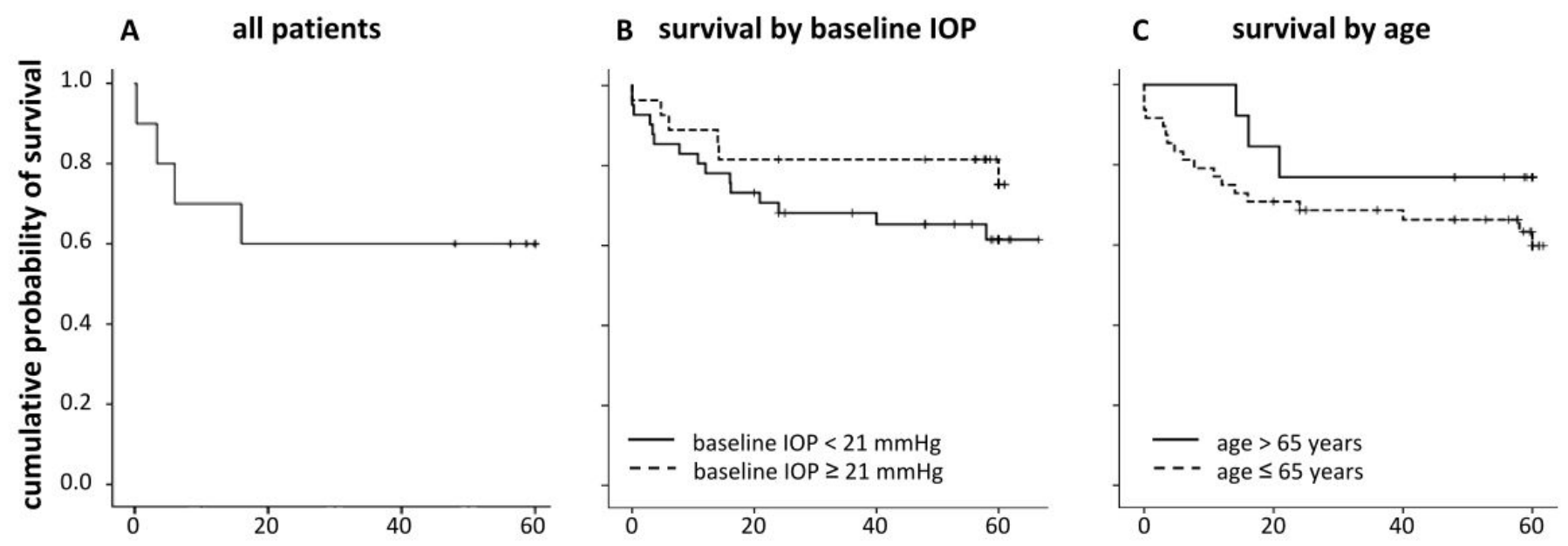

Figure 1: Kaplan-Meier survival plots with success defined as a final IOP of $\leq 21 \mathrm{mmHg}$ and a $20 \%$ reduction from baseline. A) Plot for all eyes. B) Preoperative intraocular pressure of $21 \mathrm{mmHg}$ or higher versus less than $21 \mathrm{mmHg}$. C) Age 65 or older versus younger than 65 years. 


\section{Figure 2}

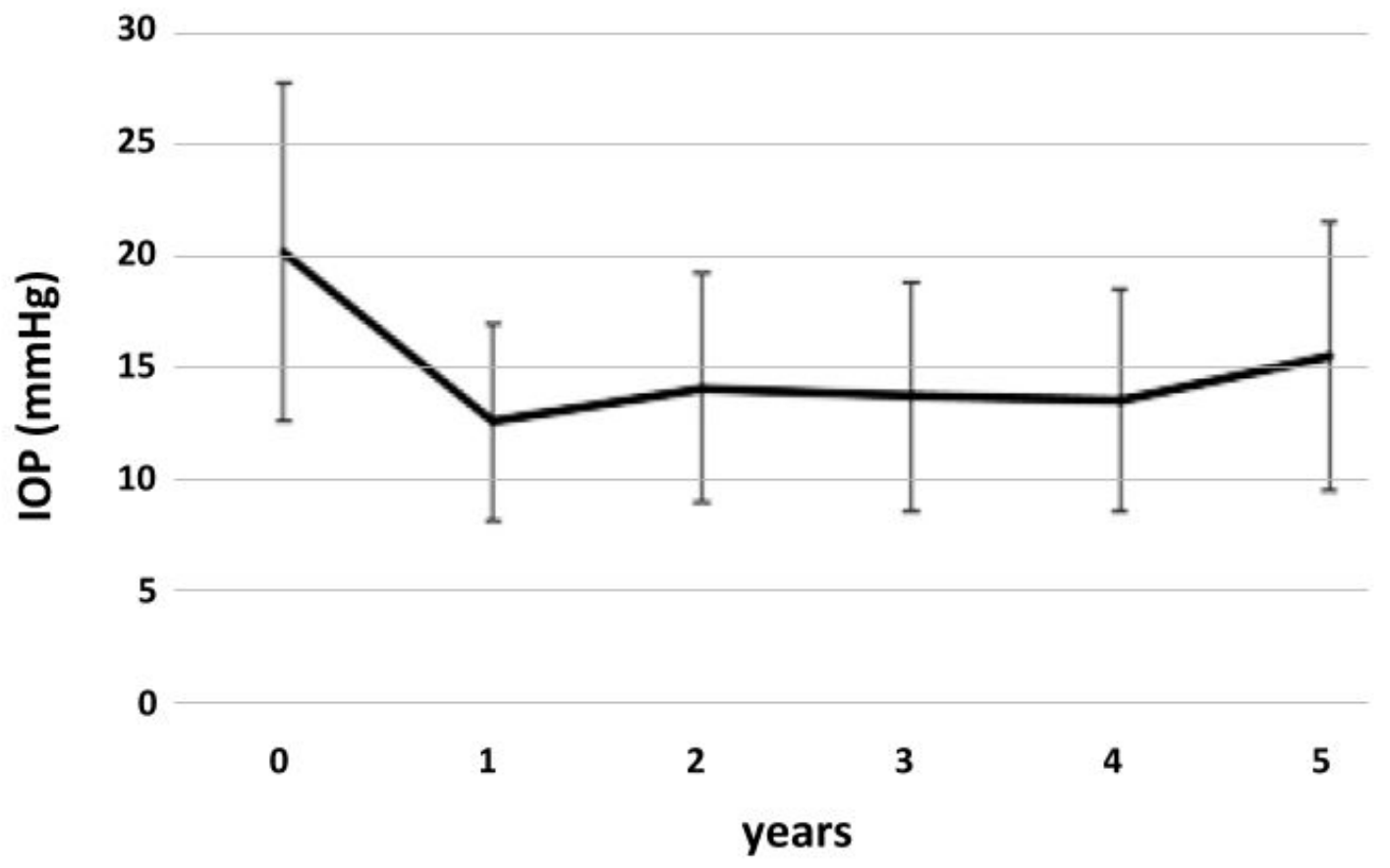

Figure 2: Preoperative and postoperative mean IOP within five years follow up. 


\section{References}

1. Minckler D, Baerveldt G, Ramirez MA, et al (2006) Clinical results with the Trabectome, a novel surgical device for treatment of open-angle glaucoma. Trans Am Ophthalmol Soc 104:40-50

2. Kaplowitz K, Schuman JS, Loewen NA (2014) Techniques and outcomes of minimally invasive trabecular ablation and bypass surgery. Br J Ophthalmol 98:579-585

3. Fallano K, Bussel I, Kagemann L, et al (2017) Training strategies and outcomes of $a b$ interno trabeculectomy with the trabectome. F1000Res 6:67

4. Tojo N, Abe S, Miyakoshi M, Hayashi A (2017) Comparison of intraocular pressure fluctuations before and after ab interno trabeculectomy in pseudoexfoliation glaucoma patients. Clin Ophthalmol 11:1667-1675

5. Kaplowitz K, Loewen NA (2015) Trabectome-Mediated Ab Interno Trabeculectomy for Secondary Glaucoma or as a Secondary Procedure. In: Advanced Glaucoma Surgery. Springer, Cham, pp 15-29

6. Akil H, Chopra V, Huang A, et al (2016) Clinical results of ab interno trabeculotomy using the Trabectome in patients with pigmentary glaucoma compared to primary open angle glaucoma. Clin Experiment Ophthalmol 44:563-569

7. Dang Y, Kaplowitz K, Parikh HA, et al (2016) Steroid-induced glaucoma treated with trabecular ablation in a matched comparison with primary open-angle glaucoma. Clin Experiment Ophthalmol 44:783-788

8. Esfandiari H, Hassanpour K, Yaseri M, Loewen NA (2018) Extended pharmacological miosis is superfluous after glaucoma angle surgery: A retrospective study. F1000Res 7: . doi: 10.12688/f1000research.13756.1

9. Bussel II, Kaplowitz K, Schuman JS, et al (2015) Outcomes of ab interno trabeculectomy with the trabectome by degree of angle opening. Br J Ophthalmol 99:914-919

10. Bussel II, Kaplowitz K, Schuman JS, et al (2014) Outcomes of ab interno trabeculectomy with the trabectome after failed trabeculectomy. Br J Ophthalmol 99:258-262

11. Mosaed S, Chak G, Haider A, et al (2015) Results of Trabectome Surgery Following Failed Glaucoma Tube Shunt Implantation: Cohort Study. Medicine 94:e1045

12. Francis BA, Minckler D, Dustin L, et al (2008) Combined cataract extraction and trabeculotomy by the internal approach for coexisting cataract and open-angle glaucoma: initial results. J Cataract Refract Surg 34:1096-1103

13. Minckler D, Mosaed S, Dustin L, et al (2008) Trabectome (trabeculectomy-internal approach): additional experience and extended follow-up. Trans Am Ophthalmol Soc 106:149-59; discussion 159-60

14. Mosaed S (2014) The First Decade of Global Trabectome Outcomes. European Ophthalmic Review 08:113-119

15. Fewtrell MS, Kennedy K, Singhal A, et al (2008) How much loss to follow-up is acceptable in long-term randomised trials and prospective studies? Arch Dis Child 93:458-461

16. Parikh HA, Bussel II, Schuman JS, et al (2016) Coarsened Exact Matching of Phaco-Trabectome to Trabectome in Phakic Patients: Lack of Additional Pressure Reduction from Phacoemulsification. PLoS One 11:e0149384

17. Neiweem AE, Bussel II, Schuman JS, et al (2016) Glaucoma Surgery Calculator: Limited Additive Effect of Phacoemulsification on Intraocular Pressure in Ab Interno Trabeculectomy. PLoS One 11:e0153585

18. (1994) Advanced Glaucoma Intervention Study. 2. Visual field test scoring and reliability. Ophthalmology 
101:1445-1455

19. Esfandiari H, Hassanpour K, Knowlton P, et al (2017) Trabectome Surgery Combined with Baerveldt Glaucoma Implantation Negates Tube Fenestration

20. Dang Y, Waxman S, Wang C, et al (2017) Rapid learning curve assessment in an ex vivo training system for microincisional glaucoma surgery. Sci Rep 7:1605

21. Jea SY, Mosaed S, Vold SD, Rhee DJ (2012) Effect of a failed trabectome on subsequent trabeculectomy. J Glaucoma 21:71-75

22. Kaplowitz K, Bussel II, Honkanen R, et al (2016) Review and meta-analysis of ab-interno trabeculectomy outcomes. Br J Ophthalmol 100:594-600

23. Luebke J, Boehringer D, Neuburger M, et al (2015) Refractive and visual outcomes after combined cataract and trabectome surgery: a report on the possible influences of combining cataract and trabectome surgery on refractive and visual outcomes. Graefes Arch Clin Exp Ophthalmol 253:419-423

24. Ahuja Y, Ma Khin Pyi S, Malihi M, et al (2013) Clinical results of ab interno trabeculotomy using the trabectome for open-angle glaucoma: the Mayo Clinic series in Rochester, Minnesota. Am J Ophthalmol 156:927-935.e2

25. Jordan JF, Wecker T, van Oterendorp C, et al (2013) Trabectome surgery for primary and secondary open angle glaucomas. Graefes Arch Clin Exp Ophthalmol 251:2753-2760

26. Widder RA, Dinslage $S$, Rosentreter A, et al (2014) A new surgical triple procedure in pseudoexfoliation glaucoma using cataract surgery, Trabectome, and trabecular aspiration. Graefes Arch Clin Exp Ophthalmol 252:1971-1975

27. Okeke CO, Miller-Ellis E, Rojas M, Trabectome Study Group (2017) Trabectome success factors. Medicine 96:e7061

28. Ngai P, Kim G, Chak G, et al (2016) Outcome of primary trabeculotomy ab interno (Trabectome) surgery in patients with steroid-induced glaucoma. Medicine 95:e5383

29. Chang SW, Tsai IL, Hu FR, et al (2001) The cornea in young myopic adults. Br J Ophthalmol 85:916-920

30. van Alphen GW (1986) Choroidal stress and emmetropization. Vision Res 26:723-734

31. Goss DA, Van Veen HG, Rainey BB, Feng B (1997) Ocular components measured by keratometry, phakometry, and ultrasonography in emmetropic and myopic optometry students. Optom Vis Sci 74:489-495

32. Kotecha A, Elsheikh A, Roberts CR, et al (2006) Corneal thickness- and age-related biomechanical properties of the cornea measured with the ocular response analyzer. Invest Ophthalmol Vis Sci 47:5337-5347

33. de Kater AW, Shahsafaei A, Epstein DL (1992) Localization of smooth muscle and nonmuscle actin isoforms in the human aqueous outflow pathway. Invest Ophthalmol Vis Sci 33:424-429

34. Gonzalez JM Jr, Ko MK, Hong Y-K, et al (2017) Deep tissue analysis of distal aqueous drainage structures and contractile features. Sci Rep 7:17071

35. Waxman S, Loewen RT, Dang Y, et al (2018) High-Resolution, Three Dimensional Reconstruction of the Outflow Tract Demonstrates Segmental Differences in Cleared Eyes. Invest Ophthalmol Vis Sci. doi:

10.13140/RG.2.2.27838.18243 